\title{
On a flexible multibody modelling approach using FE-based contact formulation for describing gear transmission error
}

\author{
Y. Benaïcha ${ }^{a, b, *}$, J. Perret-Liaudet ${ }^{a}$, J-D. Beley ${ }^{b}$,E. Rigaud ${ }^{a}$ and F. Thouverez ${ }^{a}$ \\ ${ }^{a}$ Univ Lyon, Ecole Centrale de Lyon, ENISE, ENTPE, CNRS, Laboratoire de Tribologie et Dynamique des Systèmes LTDS, UMR 5513, F-69134, \\ Ecully, France \\ ${ }^{b}$ ANSYS SAS, 35-37 rue Louis Guérin, 69100 Villeurbanne Cedex, France
}

\section{ARTICLE INFO}

\section{Keywords:}

Gear transmission error

Flexible multibody modelling

Manufacturing errors

Time varying mesh stiffness

Contact Formulation

\begin{abstract}
A B S T R ACT
This paper provides an insight into the efficiency and accuracy of a multibody approach to model gear transmission error. The multibody model is based on an augmented Lagrangian contact formulation considering a surface-to-surface contact detection. The case of spur and helical gears transmitting power between parallel shafts is considered. The static transmission error is computed without any assumptions about the contact lines positions and orientations. Tooth and wheel body flexibility and tooth profile deviations are taken into account. The main objective of this study is to evaluate the efficiency and possibilities of the proposed methodology. To this end, the static transmission error is benchmarked against the results obtained from a classical approach which is based on the computation of the equation describing the static equilibrium of the gear pair for a set of successive positions of the driving wheel.
\end{abstract}

\section{Introduction}

Noise, vibration and harshness (NVH) prediction is one of the main items in the specifications of gear transmission systems. Generally, the whining noise constitutes the dominant source of noise $[4,26,31,46]$. This structure borne noise results from the dynamic mesh force, corresponding to the time varying normal contact force. Vibrations propagate through the gear wheel bodies, the shafts and the bearings to the housing. The vibratory state of the latter is responsible for the radiated noise $[4,26]$. For example, in transportation industry, the whining noise corresponds to a large part of the noise perceived by passengers as well as the noise perceived outside the vehicle. In this context, one common goal to improve NVH is to obtain quiet gear systems by reducing the whining noise level. The dynamic mesh force results mainly from the well-known static transmission error (STE) defined as the difference between the actual position of the output gear and the position it would occupy if the gear pair were perfectly conjugate [14, 46]. The STE is expressed along the line of action as:

$$
\delta\left(\theta_{1}\right)=R_{b 2} \theta_{2}-R_{b 1} \theta_{1}
$$

$\theta_{1}$ and $\theta_{2}$ are respectively the angular position of the input wheel and the output gear. $R_{b 1}$ and $R_{b 2}$ are the base radii of the input wheel and the output gear. The gear dynamics induced by the STE remains complex to model as difficulties stem from the two following main reasons. The first one corresponds to the different space scales involved in the gear modelling $[42,10]$. The second reason concerns the coupling between the global behaviour of the whole transmission and the local behaviour at the gear mesh [20]. As a first necessary step, many studies focus on the optimization of gear macro and micro-geometries to reduce unintended vibroacoustic phenomena [3, 11, 22, 23, 35, 39] but noise reduction remains a challenge. The accurate estimation of the STE is still a major task for the NVH prediction of geared transmissions.

The static transmission error and the mesh contact force distribution along the contact line strongly depend on manufacturing errors (eccentricity, pitch error, profile error), micro-geometry deviation (longitudinal crowning, profile correction) $[14,20,17,54]$, tooth deformation (hertzian contact, tooth bending and shearing), and global deformations which induce shafts misalignment [37]). In general, a preliminary analysis uncoupled from the teeth contact computes the global deformation leading to the shafts misalignment as an input for the STE computation. In fact, it is added

\footnotetext{
*Corresponding author

youness.benaicha@ec-lyon.fr(Y.Benaïcha)

ORCID(s):
} 
to the micro-geometry deviations resulting from tooth profile corrections and manufacturing errors. Notice that these micro-geometry deviations are of the same order of magnitude as tooth and wheel body deformations. Solving the mesh contact force distribution along the contact line is thus nonlinear with the transmitted load.

Another important element for the vibroacoustic behaviour of a gear system is the internal parametric excitation source generated by the meshing stiffness fluctuation. Indeed, the peak-to-peak amplitude of its periodic variation at the meshing frequency is large. It can reach $65 \%$ of its mean value for spur gears, and $40 \%$ for helical gears. This internal excitation is deduced from the STE computation as it consists on the slope of the function of the transmitted force versus the STE. This characteristic results from a linear approximation of the transmitted mesh force function. The mesh stiffness is thus expressed as:

$$
k\left(\theta_{1}\right)=\frac{\partial F}{\partial \delta\left(\theta_{1}\right)}
$$

In classical approaches, the contact problem is solved off-line of the finite element analysis including the gear transmission. The contact lines are assumed to be located in the theoretical action plane and the gear static equilibrium is then modelled by separating the gear compliance and the local contact equations along the contact lines [24, 1, 2, 30]. Thus, the gear tooth compliance is preliminarily computed by a finite element analysis or by some alternative analytical tooth bending models using for example Ritz-Galerkin interpolation [11]. Generally, the nonlinear hertzian-like deformation is independently introduced either with an exact or approximate formulation. Furthermore, the potential contact lines located on the theoretical action plane are discretized in small segments where constant punctual forces are applied. Considering the driving angle $\theta_{1}$, a symmetric semi-positive compliance matrix $\mathbf{H}\left(\theta_{1}\right)$ is introduced for representing the relation between force and displacement at each discretized segment. An initial gap vector, here referred to as $\mathbf{e}\left(\theta_{1}\right)$, describing the initial distance between the teeth on each segment is introduced in order to take into account the tooth flank modifications and manufacturing errors. The misalignment between shafts and the deviation between teeth, induced by the global deformation of the entire transmission, are introduced at this stage. As the static transmission error, noted $\delta\left(\theta_{1}\right)$, fluctuates with the driving angle $\theta_{1}$ for a given transmitted normal load $F$, input data (matrix $\mathbf{H}\left(\theta_{1}\right)$, vector $\mathbf{e}\left(\theta_{1}\right)$ ) are iterated for successive position $\theta_{1}$, most of the time along a meshing period, involving kinematic analysis of the meshing process. For each position $\theta_{1}$, the matrix equation representing the mesh contact conditions can be formulated as the following problem:

$$
\left\{\begin{array}{l}
\mathbf{H}\left(\theta_{1}\right) \cdot \mathbf{p}\left(\theta_{1}\right)=\delta\left(\theta_{1}\right) \cdot \mathbf{1}-\mathbf{e}\left(\theta_{1}\right) \\
\mathbf{1}^{T} \cdot \mathbf{p}\left(\theta_{1}\right)=F
\end{array}\right.
$$

under the following constraints:

$$
\left\{\begin{array}{l}
\sum_{j} H_{j}\left(\theta_{1}\right) p_{j}\left(\theta_{1}\right)+\delta\left(\theta_{1}\right) \geq e_{j}\left(\theta_{1}\right) \\
p_{j} \geq 0
\end{array}\right.
$$

In this equation under constraints, the unity column vector $\mathbf{1}$ has all its components equal to 1 , the column vector $\mathbf{p}$ is the unknown distributed load to be solved and the scalar function $\delta\left(\theta_{1}\right)$ represents the unknown STE, also to be solved. Different algorithms can be used to obtain the solution $\left(\mathbf{p}\left(\theta_{1}\right)\right.$, and $\left.\delta\left(\theta_{1}\right)\right)$, the most popular one is based on a modified simplex method [9]. The mesh stiffness is computed by a numerical derivation of the transmitted load $F$ relative to the STE. These classical approaches are well adapted for cylindrical gears with parallel shafts but are more complex to implement in the case of other geometries as, for example, spiral bevel gears.

Alternatively, Vijayakar [44] combines Finite Element and surface integral methods for solving the analytical contact and the FE solution at surfaces. The finite element model predicts the deformation far away from the contact area but in the contact zone a surface integral form of Boussinesq solution is used to estimate the relative displacement of body points. Then, a "matching interface" is used to combine the finite element solution and the surface integral solution. However, the approach introduced by Vijayakar predicts the size of the contact area assuming an elliptic area as claimed by Hertz theory and this is usually not the case due to the variation of the curvatures at the contact surfaces.

Other authors $[52,7,8,51]$ compute the gear mesh stiffness by using the potential energy method. This method takes into account the bending energy, axial compressive energy, shear energy and hertzian energy. The gear tooth is modelled as a non-uniform cantilever beam and this affects the accuracy of the stiffness estimation.

After selecting one of the different strategy presented above, the static transmission error and the time varying mesh stiffness are introduced as an internal excitation in order to compute the gear dynamic response [15, 45, 28, 27]. An accurate prediction of the STE and the mesh stiffness are thus essential to compute precisely the dynamic response. 
We can also point out that mechanical transmission design in aeronautics and automotive industry is ruled by a search of weigh reduction and it often leads to wheel bodies with a thin rim or holes. Usually, a torsional stiffness to model the gear body flexibility is added but it cannot take into account possible twist of the tooth flank in the plane of action.

In this context, the approach considered here is to directly perform a flexible multibody finite element (FE) analysis of the gear pair, in order to evaluate the static transmission error taking into account the nonlinear behaviour induced by the contact between gear teeth. Different strategies to solve the meshing teeth contact through the ANSYS Mechanical ( $)$ solver are considered. Considering a flexible FE analysis to model and simulate gears is now achievable thanks to an increase in computer resources.

The paper is structured as follows: section 2 describes the FE-based contact formulation used through ANSYS Mechanical @ solver and discusses the use of an augmented lagrangian formalism with a surface-to-surface contact detection. The flexible multibody modelling is introduced in section 3. Then, the multibody strategy is assessed, by comparing the results obtained for a spur gear pair with those from a classical approach and extended to more complex gears: helical gears, spur gear with holed gear blanks, spur gear with flexible shafts and thin rim wheel.

\section{FE-based contact through ANSYS Mechanical ${ }^{\circledR}$ solver}

Solving non-linear problems by finite element analysis remains a challenge due to the contact and the nonsmooth mechanics induced by possible contact losses [50, 48, 49,47]. The contact formulation needs an accurate contact detection algorithm and an appropriate time integration scheme to deal with a large stiffness variation during the contact treatment. Thus, two particular conflicts have to be addressed for describing properly a FE-based contact, corresponding to the increased difficulty in the implementation compared with a classical FE analysis and the large number of degrees of freedom involved associated with the refinement of the mesh needed in the contact zone.

\subsection{Contact element definition}

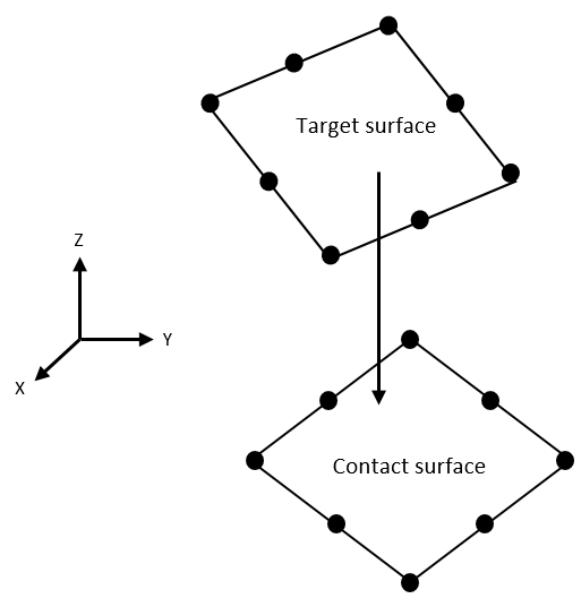

Figure 1: 8 nodes surface-to-surface contact element.

In a general contact analysis, the size and form of the contact area is not known in advance and detecting accurately bodies' interaction is essential for an efficient contact analysis. In the case of gears, tooth profile deviations are of the order of a few micrometers which requires a very fine mesh element size of the tooth surface. Thus, the contact detection has to be extremely precise. As shown in Fig. 1, a 8-node contact element intended for 3D geometries and flexible-flexible contact analysis is used. The surface of one body is conventionally taken as a contact surface and the other one as a target surface considering that both surfaces are associated with flexible bodies. In this study, a surface-to-surface contact element is privileged in order to take account of the tooth micro-geometry as opposed to node-to-surface element for which only target nodes are constrained to prevent penetration into the contact surface. Indeed, in this formulation, the contact conditions are introduced so that each target node interacts with a projected 
point on the contact surface. Consequently, contact conditions involve a single node and it concentrates the force at this node. So, node-to-surface facilitates unintended penetration during the contact detection and it leads to less precise stress and pressure. In contrast, the target surface can penetrate into the contact surface for the surface-to-surface element and this interpenetration tolerance has to be adjusted in regards to the tooth profile modifications. This surface is projected on the contact surface and the contact detection points are the integration points which are either nodal points or gauss points [6]. The interpenetration is handled on the target surface and its integration points along the normal direction [5]. The surface-to-surface element involves more nodes during the contact treatment which has a smooth effect on the stress and the pressure.

\subsection{Frictionless contact formulation}

Several contact algorithms are associated with the previous contact element. The pure penalty method, augmented lagrangian method and pure lagrange multiplier method are largely used in FE softwares and particularly in the ANSYS Mechanical ${ }^{\circledR}$ solver. In this paper, we focus on frictionless contact because vibroacoustic phenomena in gear transmission are mainly caused by the fluctuation of the normal contact force at the gear pair $[4,32]$. If one is interested in power losses, friction and therefore tangential contact forces, should be considered [19, 43].

Contact formulation can be classified into two categories corresponding to penalty methods and lagrangian methods. The pure penalty method received a larger approval in the literature due to its implementation convenience. It consists in adding a penalty term represented by a nonphysical (or mathematical) contact stiffness but the main drawback is that the amount of penetration between the two surfaces depends on this stiffness [12, 38, 33]. For this reason, the penalty formulation can lead to ill-conditioned problems and inaccurate solutions. The pure lagrangian method uses a lagrange multiplier as an added degree of freedom and this approach enforces zero penetration. It does not require additional stiffness but it needs more iterations to reach contact conditions convergence and therefore it is computationally demanding [29]. This method can also lead to inaccurate solutions because the method overconstrains the model and needs much more iterations to reach the convergence compared with the pure penalty method. Another method named the Augmented Lagrange Multiplier method [36, 21] has been developed to keep the advantages of the two previous contact formulations. The approach is an iterative combination of the penalty method and lagrange multiplier formulation. Compared to the penalty method, the augmented lagrangian method usually leads to better conditioning and is less sensitive to the magnitude of the contact stiffness coefficient. The impenetrability is thus achieved while improving the convergence. The augmented lagrangian formulation is retained to describe the gear contact and the nonsmooth mechanics is represented by Signorini conditions [47, 48, 49]:

$$
\left\{\begin{array}{l}
d_{i} \geq 0 \\
F_{i} \geq 0 \\
d_{i} F_{i}=0
\end{array} \quad \forall i\right.
$$

where to every contact node $i$ corresponds a gap function $d_{i}$ measuring the distance between the contact and the target surfaces along the normal direction and $F_{i}$ is the contact force acting on the target surface defined by:

$$
F_{i}^{j}=\kappa d_{i}^{j}+\lambda_{i}^{j}
$$

$\kappa$ is the mathematical stiffness coefficient, $\lambda_{i}^{j}$ is the iterative lagrange multiplier associated to the contact node $i$ at the iteration $j$ which is updated until $d_{i}^{j}$ is sufficiently small $[21,36]$.

\section{Problem formulation}

\subsection{Gear geometry definition}

In the study, we focus on spur and helical gears transmitting power between parallel shafts. The tooth geometry definition received a particular attention. As mentioned in the introduction, tooth profile deviation from the theoretical involute tooth profile is of the order of a few micrometers. Therefore an accurate geometry generation is needed. Indeed, the generation is performed using high order Non-Uniform Rational Basis Splines with a high control point number. It is shown in $[34,40]$ that Non-Uniform Rational Basis Splines lead to better accuracy for contact problems without considering profile deviation. 
Here, we introduced two types of tooth deviations from the theoretical involute tooth profile: a longitudinal crowning (see Fig. 2b) and a linear tip relief (see Fig. 2a). They are intentional removal of material from gear tooth flanks. They help reducing dynamic loadings and compensate shafts misalignment and deflections. Longitudinal crowning is generally introduced to maintain the contact at the tooth flank centre and linear tip relief smoothens the meshing transition process.

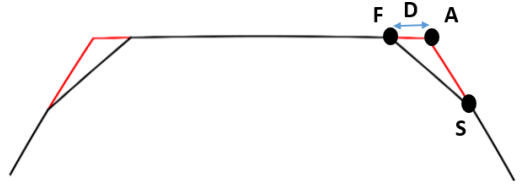

(a)

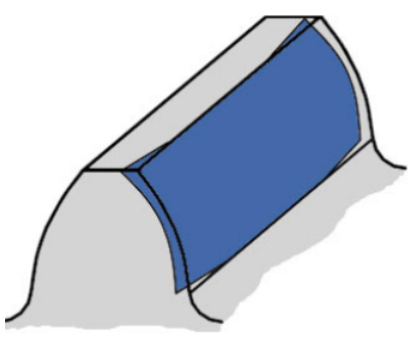

(b)

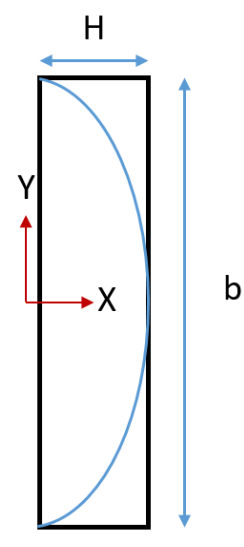

(c)

Figure 2: Description of linear tip relief deviation (a) where $S$ is the starting point, $D$ is an amount of profile modification at the tooth tip starting for the point $A$ to the point $F$. Longitudinal crowning modification (b) and parameters description from the top (c). $H$ is the maximum depth of crowning. $y$ is the varying depth parameter covering the tooth width $b$.

\subsection{Multibody gear simulation}

The problem to solve the gear dynamics is expressed as follow:

$$
\mathbf{M} \ddot{\mathbf{x}}+\mathbf{C} \dot{\mathbf{x}}+\mathbf{K x}+\mathbf{F}_{n l}(\mathbf{x})=\mathbf{F}_{\text {ext }}
$$

where $\mathbf{x}$ contains the generalised displacement of each degree-of-freedom and $\mathbf{M}, \mathbf{C}, \mathbf{K}$ are respectively the mass, damping and stiffness matrix. $\mathbf{F}_{n l}$ is the contact force and $\mathbf{F}_{\text {ext }}$ is the vector of external forcing. The approach presented here is focusing on the quasi-static behaviour of the gear pair. Indeed, the dynamic equation (7) is solved for a slow rotation of the driving gear $(1 \mathrm{rpm})$ and the dynamical effects are needed only to improve the convergence during the gap covering.

It is a scientific progress to deal directly with flexible multibody analysis in quasi-static operating conditions for evaluating the transmission error, especially to show complex effects of the gear mesh process. Indeed, the contact treatment phase (contact detection and contact force computation) is solved in real operating conditions. So, specific gear mesh behaviour is brought to light compared to classical methods. The multibody modelling approach aims at providing a generic model that can be used as a reference for meshing settings, contact settings and loading conditions. The proposed methodology intends to accurately identify the gear internal excitation. For this purpose, the procedure, the implementation and the results precision are evaluated. As mentioned in the previous section, a fine mesh is of paramount importance to highlight the influence of the load level but also to take into account the hertzian deformation and the microgeometry. Knowing that the tooth deformation takes account of tooth deflection and local hertzian-like deformation induced by the contact, the mesh has to be refined accordingly in the contact area. Moreover, a nonlinear analysis must be carried out for various levels of output torque to show the effect of load on the tooth deformation. To achieve the simulation, three steps have to be set up:

- firstly, the gear backlash is covered by a rotation of the driving wheel in order to establish the contact at an initial state.

- Then, the output torque is applied to the driven wheel while maintaining the driving wheel. 
- Finally, a rotation of the driving wheel is performed over a period corresponding to the fundamental period of static transmission error (at least one mesh period).

A control node is defined at each gear centre to apply boundary conditions and to measure the time varying input and output gear angles. The static transmission error $\delta\left(\theta_{1}\right)$ is then computed along the line of action and the mesh stiffness $k\left(\theta_{1}\right)$ is approximated by a numerical differentiation of the transmitted load $F$ versus $\delta\left(\theta_{1}\right)$. The discretization of the angular position of the driving wheel has to be finely defined for describing properly the sudden variation of the STE. At least 40 points over one mesh period are needed. In addition, to avoid numerical errors, the differentiation of the mesh stiffness is achieved by taking a centred differentiation step equal to $15 \%$ of the load and the STE.

\section{Results and discussion}

\subsection{Preliminary validations}

This section is a preliminary analysis of the proposed methodology in order to optimally compute the static transmission error. Firstly, the influence of the mesh on the STE is evaluated. Then, the time cost is reduced through an optimal selection of computer resources.

\subsubsection{Mesh convergence}

To propose an efficient multibody simulation, a convergence study on the FE mesh along the tooth was carried out. The involute profile is divided into 50,70 and 100 hexa-linear elements, and an inflation of 30 layers is added to detect edge effects and hertzian-like deformation. The mesh element size in the contact area is respectively $70 \mu \mathrm{m}, 50 \mu \mathrm{m}$ and $38 \mu \mathrm{m}$. The procedure is applied for validation on a spur gear pair considering the following gear characteristics (Table 1):

\begin{tabular}{ccccc}
\hline Name & Designation & Gear 1 & Gear 2 & Unit \\
\hline Module & $m$ & \multicolumn{2}{c}{2} & $\mathrm{~mm}$ \\
Number of teeth & $Z$ & 50 & 50 & - \\
Pressure angle & $\alpha$ & \multicolumn{2}{c}{20} & $\mathrm{deg}$ \\
Base radius & $r_{b}$ & 46.984 & 46.984 & $\mathrm{~mm}$ \\
Profile shift coefficient & $x$ & 0 & 0 & - \\
Addendum coefficient & $h_{a}$ & 1 & 1 & - \\
Dedendum coefficient & $h_{d}$ & 1.25 & 1.25 & - \\
Face width & $b_{f}$ & 20 & 20 & $\mathrm{~mm}$ \\
\hline Tip relief modification & & & & \\
\hline Starting radius & $r_{S}$ & 50.26 & 50.26 & $\mathrm{~mm}$ \\
Depth & $D$ & 5 & 5 & $\mu \mathrm{m}$ \\
\hline Longitudinal crowning modification & & & & \\
\hline Maximum depth & $H$ & 0 & 0 & $\mu \mathrm{m}$ \\
\hline
\end{tabular}

Table 1: Characteristics of the spur gear

The mesh generation with about 700000 nodes and about 2 million degrees-of-freedom is presented in Fig. 3 with a close-up (see Fig. 3b) in the teeth region. The nonlinear quasi-static analysis is carried out for a low output torque $T=0.5 \mathrm{~N}$.m, especially for evaluating the influence of the mesh size on the transmission error. The associated unloaded STE is presented in Fig. 4 for different levels of detail of the tooth mesh. The shape of the curves follows the long tip relief of $5 \mu \mathrm{m}$ only for an element size lower than $50 \mu \mathrm{m}$. The comparison of the unloaded transmission error exhibits the importance of dealing with a specific mesh. 70 divisions in the involute profile are retained in the following sections. 


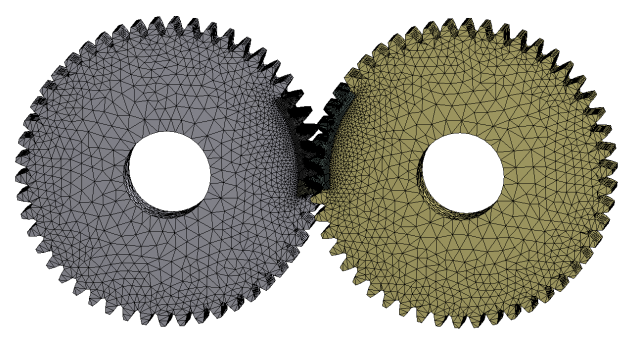

(a)

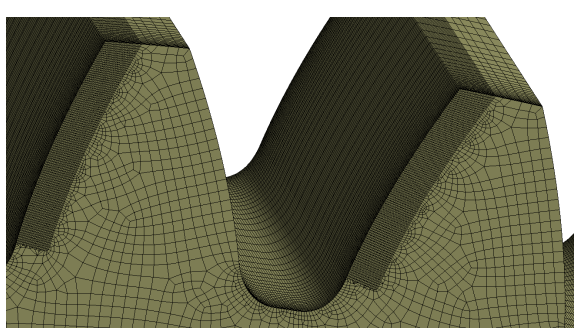

(b)

Figure 3: (a) the spur gear mesh with hexa-linear element at the tooth and tetra element elsewhere (b) close-up of the tooth mesh with 100 hexa-linear element and an inflation.

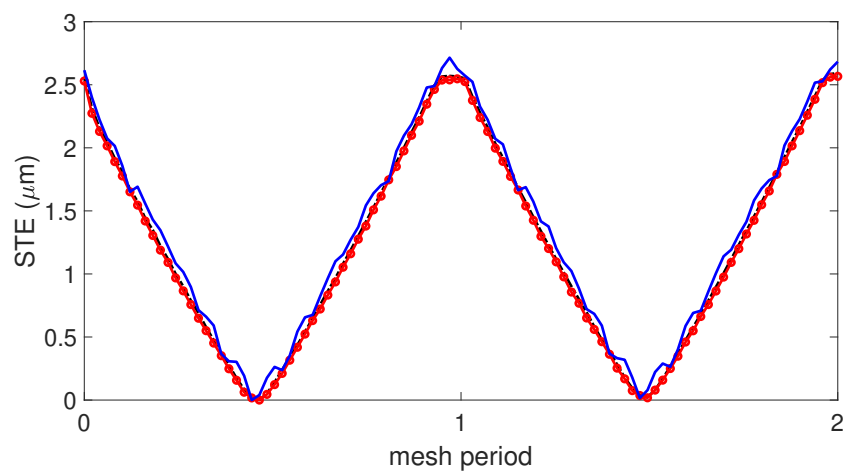

Figure 4: Unloaded transmission error for coarse mesh (50 divisions, - $)$ ), intermediate mesh (70 divisions, $\bullet$ ) and fine mesh (100 divisions, - - )

\subsubsection{Time cost}

The Guyan condensation [13] is largely used in solid mechanics for solving the static analysis of contacting structures. The approach provides an exact solution of nonlinear problems while improving the time cost compared with a direct solving. The idea is to consider two sets of degrees of freedom (DOF), one for active $u_{A}$ (contact nodes here) and another complementary $u_{C}$. The DOF separation allows the identification of a linear relationship between the two sets of DOF:

$$
\mathbf{u}_{C}=-\mathbf{K}_{C C}^{-1} \mathbf{K}_{C A} \mathbf{u}_{A}
$$

where the finite element stiffness $K$ is split into four blocks:

$$
\left[\begin{array}{ll}
\mathbf{K}_{A A} & \mathbf{K}_{A C} \\
\mathbf{K}_{C A} & \mathbf{K}_{C C}
\end{array}\right]
$$

Considering our gear model, about 6000 contact nodes are possibly involved during the contact treatment. A refinement of the mesh at the contact interface leads to full matrices after the condensation on the nonlinear degrees of freedom. So, the matrix operation shown in the equation (8) becomes computationally demanding. To overcome this difficulty, we investigate a direct resolution of the full system using domain decomposition.

Domain decomposition is a parallel computation technique widely used in computational mechanics [18, 25, 53]. It consists in the division of the whole domain in several partitions. The mechanical problem is solved independently on each partition and an update of the boundaries enables communication between the partitions. Herein, we apply a domain decomposition on a cluster using CPU resources ( 1 computer node $=16$ cores, $2.6 \mathrm{GHz}$ and 64 Go RAM) in order to achieve the flexible multibody simulation. A parametric study on the case presented in Table 2 is conducted by increasing the CPU resources from 16 cores ( 1 computer node) to 96 (6 computer nodes). The simulations are benchmarked against the Guyan method. 
On a flexible multibody modelling approach using FE-based contact formulation for describing gear transmission error

\begin{tabular}{cccc}
\hline Simulations & Time cost (s) & Time reduction $(\%)$ & Number of cores \\
\hline REF (Guyan) & 144000 & - & - \\
1 & 53224 & $63 \%$ & 16 \\
2 & 18303 & $87 \%$ & 32 \\
3 & 15286 & $89 \%$ & 64 \\
4 & 19226 & $86 \%$ & 96
\end{tabular}

Table 2: Parametric study on spur gear to reduce the time cost

Figures in Table 2 show that 64 cores is the optimal choice for solving the nonlinear analysis. Constantly increasing the domain discretization is not leading to a proportional constant time reduction. Indeed, for 96 cores the time reduction (86\%) is less than for 64 cores. This is induced by the large number of information transfers needed to communicate with each partition. The multibody analysis is thus performed in the following sections by solving the full nonlinear system using 64 cores.

\subsection{Numerical comparison with a classical approach}

The objective of this section is to evaluate the main differences between the proposed multibody methodology and a classical approach. The assessment is made in terms of static transmission error and meshing stiffness fluctuations. The gear transmission presented in Table 1 is retained for the comparison. The study is performed for various output torques in order to obtain relevant comparative results. Then, the potential effects on the dynamic behaviour are discussed.

\subsubsection{Static transmission error}

Several nonlinear analysis are carried out for different output torques and a map of the static transmission error is built. Fig. 5a and Fig. 5b are respectively the maps of the STE using the multibody method and the classical approach. The gear mesh procedure is properly handled by the multibody approach and the periodicity of the STE is well represented. Fig. 5a and Fig. 5b show a good match between the transmission curves for an output torque from 0.5 N.m to 50 N.m. Indeed, the tip relief of $5 \mu \mathrm{m}$ is clearly identified by both approaches. However, for a torque level up to 50 N.m, the classical approach underestimates the mean values of the STE while the STE shapes have a smoother variation using the multibody method. In fact, the transition from one tooth pair to another is progressively handled as shown in Fig. 6. Indeed, in Fig. 5b, a sudden variation of the STE is visible for a torque equal to 50 N.m which can be explained by a brutal transition of the number of teeth pair involved during the contact treatment. This phenomenon is amplified as the torque is increased. The time instant when the number of teeth in contact changes is previously determined in the classical approach with the contact ratio of the gear pair expressed as $\epsilon_{\alpha}=g_{\alpha} / p_{b}$ while $g_{\alpha}$ is the length of path of contact and $p_{b}$ is the base pitch. This indicator explains the brutal variation of the STE.

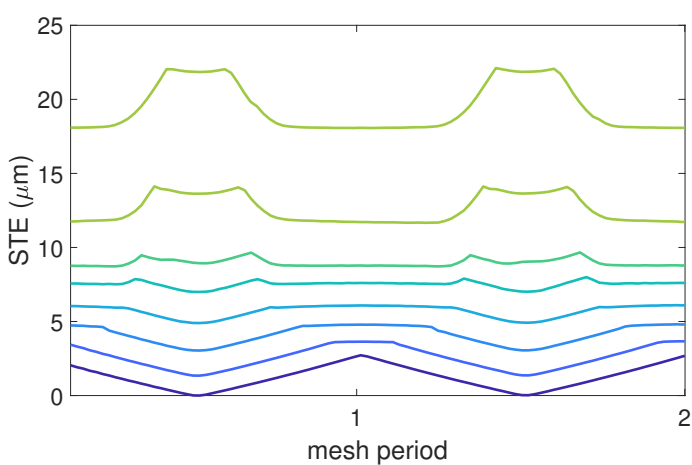

(a)

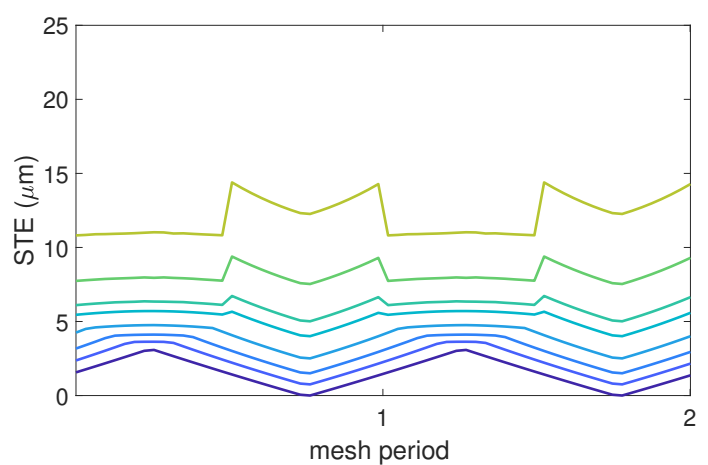

(b)

Figure 5: The static transmission error for different torque levels $T=[0.5,15,30,50,80,100,150,250] \mathrm{N}$.m and over two mesh periods. Numerical results through ANSYS Mechanical $\mathbb{R}$ solver (a) and the classical method (b). 
Moreover, the map brings to light the nonlinear variation of the STE in regards to the load. The peak to peak transmission error (PPTE) is measured from the STE map for the proposed approach (Fig. 5a) and the classical approach (Fig. 5b) in order to highlight the relation between load and deformation. The variations of the PPTE presented in Fig. 7 allow an estimation of an optimal torque. Indeed, The PPTE amplitude of the classical approach decreases until an output torque close to $T=70$ N.m and then, it increases again, whereas the same phenomena appears at $T=100$ N.m for the multibody approach. The optimal torque ranges for the classical approach and the multibody approach are respectively $[80,150]$ N.m and $[60,110]$ N.m. Differences in the estimation of the optimal torque and the mean value of the STE can be explained on the one hand by the method retained in the classical strategy for the computation of the compliance matrix $H(\theta)$ and on the other hand by the number of teeth detected during the contact treatment as mentioned above. These differences can lead to an imprecise micro-geometry optimization when using standard methods.

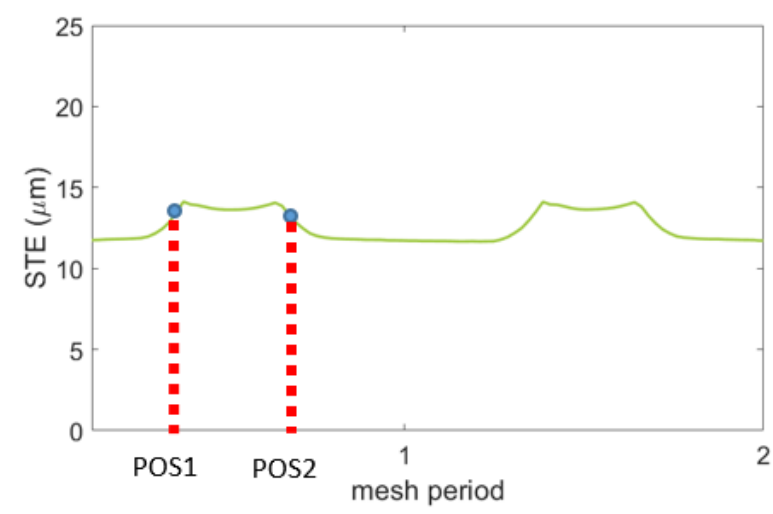

(a)

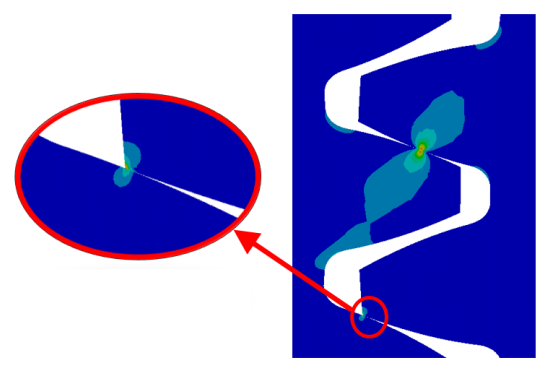

(b)

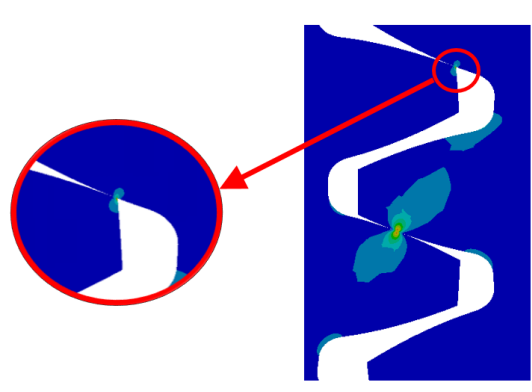

(c)

Figure 6: STE for 150 N.m with two contact positions named POS1 and POS2 (a). A close-up at the contact POS1 (b) and POS2 (c).

\subsubsection{Mesh stiffness}

The mesh stiffnesses are evaluated for the output torques $T=[0.5,15,30,50,80,100,150,250]$ N.m over two mesh periods. The mean value and the fluctuations of the mesh stiffness of the proposed methodology and the classical approach are both governed by the output torque as shown in Fig. 8. It appears three ranges of variation corresponding to: low torque $[15,50]$ N.m, medium torque $[50,100]$ N.m and high torque $[100,250]$ N.m.

The mean values of the mesh stiffnesses computed through the multibody approach are two time less than the ones obtained with the classical approach. The critical modes which are the modes for which the strain energy contribution at the mesh is maximum are governed by the mean value of the mesh stiffness. The critical frequencies of the system are thus shifted to lower frequencies by $\sqrt{2}$. This change affects the critical operating speeds of the system. In addition to this, as shown in Fig. 8, the fluctuations of the mesh stiffnesses are also two time less for the multibody method 


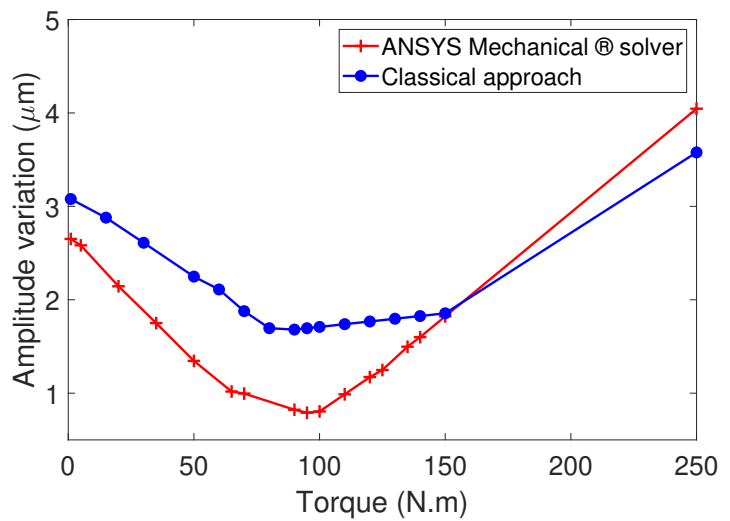

Figure 7: Comparison of the different peak to peak transmission error (a): - Classical approach, and —ANSYS Mechanical R solver

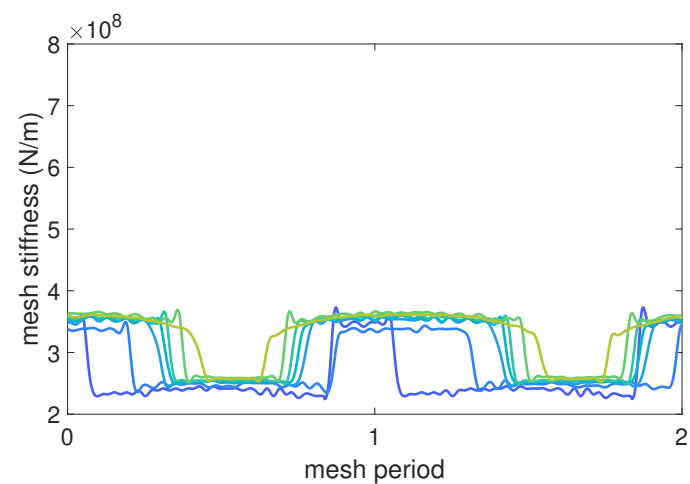

(a)

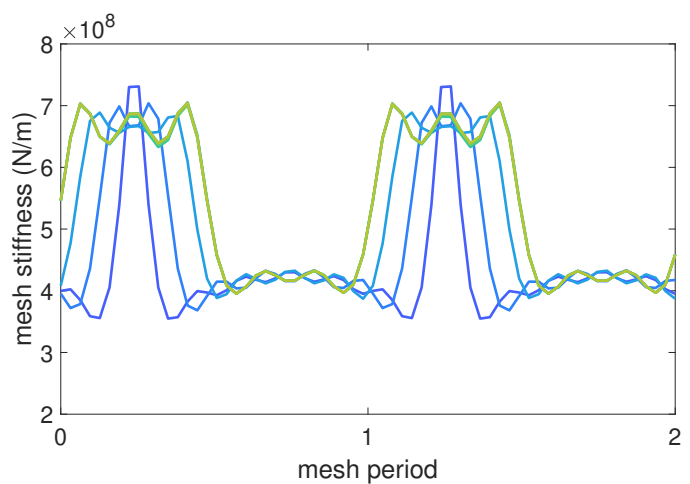

(b)

Figure 8: Mesh stiffness for different torque levels $T=[15,30,50,80,100,150,250]$ N.m: (a) ANSYS Mechanical $\mathbb{R}$ solver and (b) classical approach.

compared with the classical approach. This large variation modifies parametric phenomena and the amplitude of resonance of the dynamic response [41].

\subsubsection{Normal contact characteristics of mating gear teeth}

In solid mechanics, the local contact behaviour of structures is often modelled with Hertz theory [16]. It is an efficient way to approximate local deformations and to predict contact areas. In the classical approach, the local deformation is estimated by using a hertzian-like deformation. In constrast, the multibody approach has no assumption of the contact curvature and the methodology provides stress, contact pressure and contact area during the gear mesh. This section is devoted to comparing these physical quantities with the results predicted by Hertz theory.

The cylindrical Hertzian contact stress theory for two infinite cylinders under load is based on the following assumptions:

- surfaces are continuous and non-conforming.

- Each interacting body is considered as an elastic half-space with small strains.

- It is a frictionless contact.

- The contact areas are small compared to the size of the radii of curvature of surfaces. 
The curvature radii at the contact point of two cylinders are represented in gears by the tangential segments of the base radii connected to the contact point. For a contact point close to the pitch radius, the contact width $h$, the contact depth $z_{0}$, the maximum pressure $P_{0}$ and the maximum shear stress $\tau$ as described in Fig. 9 are compared with the multibody approach (see Table 3 ). The contact width is accurately approximated with the multibody approach. Indeed, as shown in Table 3, the approach generates $229 \mu \mathrm{m}$ of contact width and $204 \mu \mathrm{m}$ is accounted for Hertz theory. Additionally, the contact analysis converged in terms of maximum pressure and maximum shear stress as depicted in Table 3 where only $20 \mathrm{MPa}$ of differences with Hertz are measured. The results obtained with the proposed approach are thus in good agreement with the those acquired by Hertz theory. In contrast, theses physical quantities can't be retrieved directly with a classical approach. It required additional analyses.

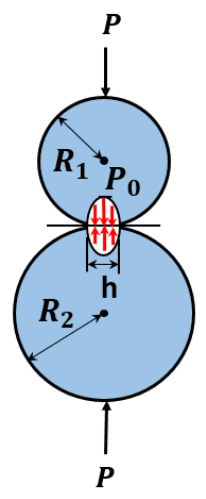

(a)

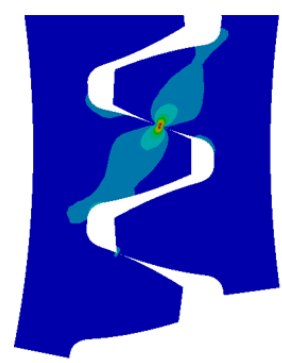

(b)

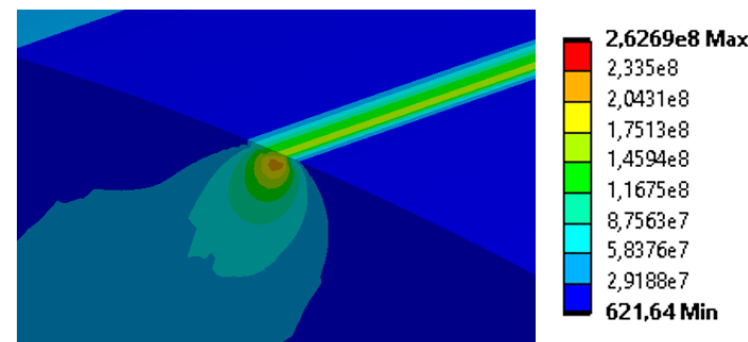

(c)

Figure 9: (a) Description of the cylindrical contact stress claimed by Hertz theory, (b) shear stress for a torque of $T=100$ N.m and (c) a close-up of the shear stress in $\mathrm{Pa}$ at the middle of the tooth width is depicted.

\begin{tabular}{cccc}
\hline Physical quantities & Hertz & Proposed approach & Unit \\
\hline$h$ & 204 & 229 & $\mu \mathrm{m}$ \\
$P_{0}$ & 663 & 643 & $\mathrm{MPa}$ \\
$\tau$ & 199 & 221 & $\mathrm{MPa}$ \\
$z_{0}$ & 79.5 & 98.3 & $\mu \mathrm{m}$
\end{tabular}

Table 3: Comparative study on spur gear with Hertz theory

\subsection{Capability of the multibody method to detect specific phenomena}

The study carried out in this section, applies the proposed multibody approach to several gear transmissions in order to show the large possibility of the method and to bring to light some phenomena. The gear pairs are classified in three test cases. The first case compares a gear pair with a longitudinal crowning to a gear pair without. Then, the effect of thin rim and flexible shaft on spur gear is evaluated. Finally, modulations in time and frequency domains of a spur gear with holed gear blanks are identified. Theses phenomena are not properly taken into account with classical approaches. To identify theses specific effects, the fluctuations of the STE for an output torque of $T=115$ N.m are thus computed, described and discussed.

\subsubsection{Effect of crowning}

The helical gear pairs are described in the Table 4 and the geometry is reported in Fig. 10. One of them include a longitudinal crowning modification. Fig. 11 displays the STE fluctuations of the helical gear pairs. The longitudinal crowning modifies the shape of the STE and it appears a larger curvature as shown in Fig. 11b. The peak to peak amplitude increases significantly until it reaches $2 \mu \mathrm{m}$ compared with the standard helical gear pair (see Fig. 11a). These effects on the STE are explained by the fact that the contact force is localized at the tooth flank center. 
On a flexible multibody modelling approach using FE-based contact formulation for describing gear transmission error

\begin{tabular}{|c|c|c|c|c|}
\hline Name & Designation & Gear 1 & Gear 2 & Unit \\
\hline Module & $m$ & & 2 & $\mathrm{~mm}$ \\
\hline Number of teeth & $Z$ & 50 & 50 & - \\
\hline Pressure angle & $\alpha$ & & 20 & deg \\
\hline Helix angle & $\beta$ & & 15 & deg \\
\hline Base radius & $r_{b}$ & 48.439 & 48.439 & $\mathrm{~mm}$ \\
\hline Profile shift coefficient & $\mathrm{x}$ & 0 & 0 & - \\
\hline Addendum coefficient & $h_{a}$ & 1 & 1 & - \\
\hline Dedendum coefficient & $h_{d}^{a}$ & 1.25 & 1.25 & - \\
\hline Face width & $b$ & 20 & 20 & $\mathrm{~mm}$ \\
\hline \multicolumn{5}{|l|}{ Tip relief modification } \\
\hline Starting radius & $r_{S}$ & 52.024 & 52.024 & $\mathrm{~mm}$ \\
\hline Depth & $D$ & 5 & 5 & $\mu \mathrm{m}$ \\
\hline \multicolumn{5}{|c|}{ Longitudinal crowning modification } \\
\hline$H$ & 10 & 10 & Maximum depth & $\mu \mathrm{m}$ \\
\hline
\end{tabular}

Table 4: Gear characteristics of the helical gear pairs

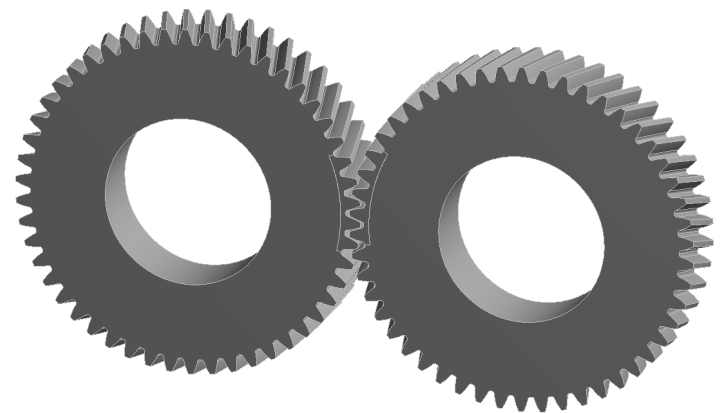

Figure 10: Helical gear pair without and with longitudinal crowning

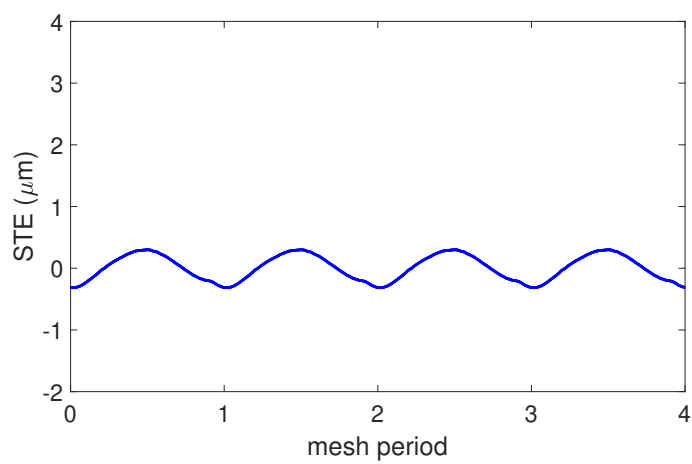

(a)

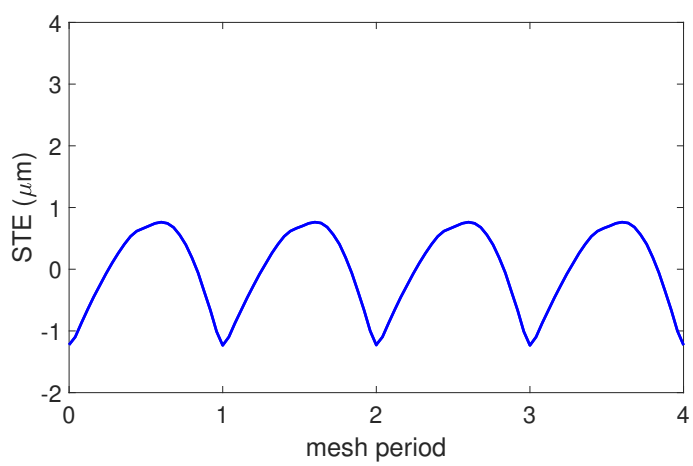

(b)

Figure 11: Fluctuation of the static transmission error over 4 mesh periods for an output torque $T=115$ N.m: (a) helical gear without longitunal crowning, (b) helical gear with longitunal crowning of $10 \mu \mathrm{m}$ 


\subsubsection{Effect of thin rim and flexible shafts}

This section analyzes the influence of a thin rim wheel and flexible shafts on the STE fluctuations. The thin rim is located in the midplane of the gears but the gears are not at the center of the shafts in order to accentuate possible rotation of the tooth flank in the plane of action. The Table 5 described in detail the micro and macro geometry and the gear pair is depicted in Fig 12. The STE fluctuations are compared with those of the spur gear presented in Fig. 7. The shape of the curve is modified and the peak to peak amplitude is increased from $1 \mu \mathrm{m}$ (see Fig. 7) to $4 \mu \mathrm{m}$ (see Fig. 13).

\begin{tabular}{ccccc}
\hline parameters & Gear 1 & Gear 2 & Nomenclature & UoM \\
\hline Module & $m$ & & 2 & $\mathrm{~mm}$ \\
Number of teeth & $Z$ & 50 & 50 & - \\
Pressure angle & $\alpha$ & & 20 & $\mathrm{deg}$ \\
Base radius & $r_{b}$ & 46.984 & 46.984 & $\mathrm{~mm}$ \\
Profile shift coefficient & $\mathrm{x}$ & 0 & 0 & - \\
Addendum coefficient & $h_{a}$ & 1 & 1 & - \\
Dedendum coefficient & $h_{d}$ & 1.25 & 1.25 & - \\
Face width & $b$ & 20 & 20 & $\mathrm{~mm}$ \\
\hline Tip relief modification & & & & \\
\hline Starting radius & $r_{S}$ & 50.26 & 50.26 & $\mathrm{~mm}$ \\
Depth & $D$ & 5 & 5 & $\mu \mathrm{m}$ \\
\hline Rim wheel dimension & & & & \\
\hline rim width & $b_{w}$ & 8 & 8 & $\mathrm{~mm}$ \\
\hline Shaft dimension & & & & \\
\hline Shaft length & $L$ & 300 & 300 & $\mathrm{~mm}$ \\
\hline
\end{tabular}

Table 5: Gear characteristics of the spur gear with thin and flexible shafts

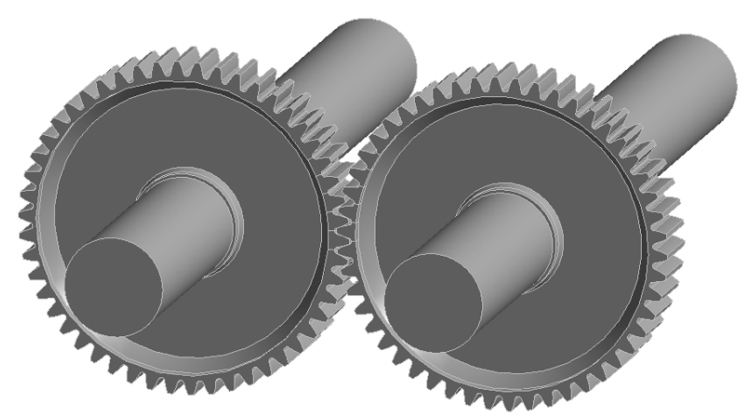

Figure 12: Spur gear with thin rim and flexible shafts named Case $C$

Moreover, the deformation of the shaft induces a misalignment as shown in Fig. 14a. Indeed, the contact pressure is partially distributed on the tooth flank when the gear teeth are in contact. This particularity is accounted in-line with the multibody approach which is not the case for a classical approach for which the contact is based on the theoretical contact lines. All couplings of the gear transmission are taken into account during the STE computation and this affects significantly the contact pressure distribution and the STE fluctuations. 


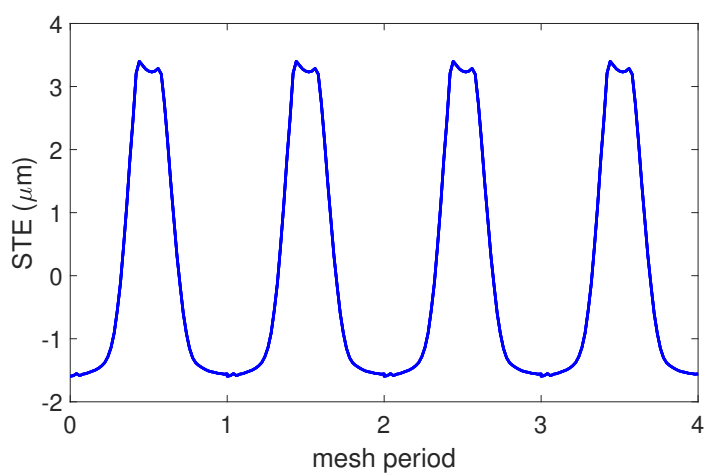

Figure 13: Fluctuation of the static transmission error over 4 mesh periods for an output torque of $115 \mathrm{~N} . \mathrm{m}$

\begin{tabular}{|l} 
9,2067e8 Max \\
$8,1837 \mathrm{e} 8$ \\
$7,1607 \mathrm{e} 8$ \\
$6,1378 \mathrm{e} 8$ \\
$5,1148 \mathrm{e} 8$ \\
$4,0919 \mathrm{e} 8$ \\
$3,0689 \mathrm{e} 8$ \\
$2,0459 \mathrm{e} 8$ \\
$1,023 \mathrm{e} 8$ \\
0 Min
\end{tabular}

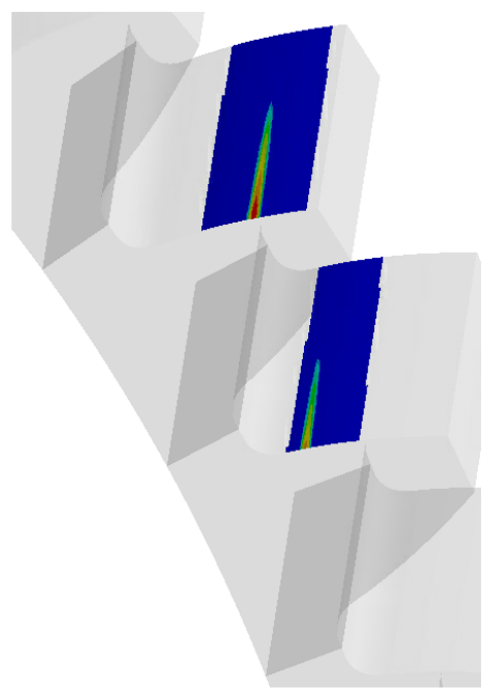

(a)

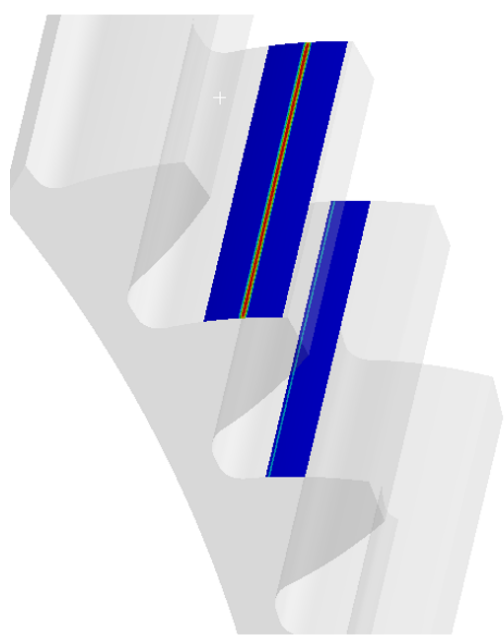

(b)

Figure 14: Contact pressure distribution on the mating teeth in $\mathrm{Pa}$ : (a) spur gear with thin rim and flexible shafts, (b) standard spur gear.

\subsubsection{Effect of gear body with holes}

This part investigates the influences of holed gear blanks on the STE fluctuations. We consider a spur gear with 8 holes on each gear bodies as shown in Fig. 15. Fig. 16 displays STE fluctuations and the frequency spectrum. Holes in gear blanks are responsible for additional harmonic components, especially at low frequency. Indeed, a harmonic corresponding to the number of holes is created and his amplitude exceeds the mesh harmonic (see Fig. 16b).

The harmonic content show also the existence of sidebands. Considering $H_{Z}$ and $H_{N_{H}}$ which are respectively the mesh harmonic and the harmonic of holes, we can identify sidebands as $H_{s b}=H_{Z} \pm H_{N_{H}}$. For example on the Fig. $16 \mathrm{~b}$ we see the harmonics $H_{42}, H_{50}$ and $H_{58}$

So, changes in gear blank topology increase the harmonic content of the STE. This phenomena is not properly handled within the state-of-the-art strategies for which the contact is established at the theoretical contact lines. 
On a flexible multibody modelling approach using FE-based contact formulation for describing gear transmission error

\begin{tabular}{ccccc}
\hline parameters & Gear 1 & Gear 2 & Nomenclature & UoM \\
\hline Module & $m$ & & 2 & \\
Number of teeth & $Z$ & 50 & 50 & $\mathrm{~mm}$ \\
Pressure angle & $\alpha$ & & 20 & - \\
Base radius & $r_{b}$ & 46.984 & 46.984 & $\mathrm{deg}$ \\
Profile shift coefficient & $x$ & 0 & 0 & - \\
Addendum coefficient & $h_{a}$ & 1 & 1 & - \\
Dedendum coefficient & $h_{d}$ & 1.25 & 1.25 & - \\
Face width & $b_{f}$ & 20 & 20 & $\mathrm{~mm}$ \\
\hline Tip relief modification & & & & \\
\hline Starting radius & $r_{S}$ & 50.26 & 50.26 & $\mathrm{~mm}$ \\
Depth & $D$ & 5 & 5 & $\mu \mathrm{m}$ \\
\hline Lightweighting & & & & \\
\hline Number of holes & $N_{H}$ & 8 & 8 & - \\
Radius of holes & $r_{H}$ & 20 & 20 & $\mathrm{~mm}$
\end{tabular}

Table 6: Gear characteristics of the spur gear with holed gear blanks

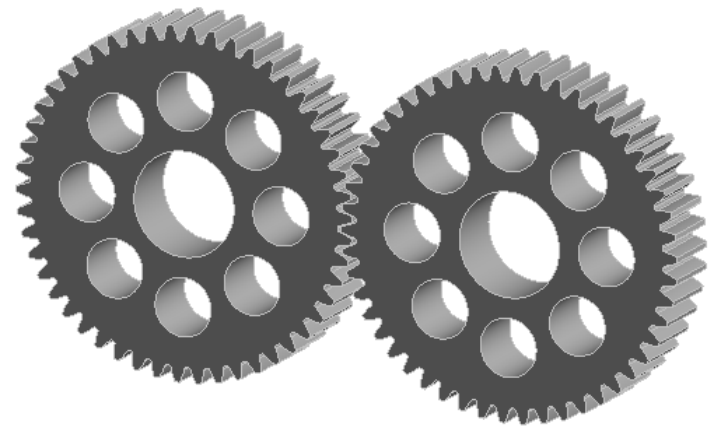

Figure 15: Spur gear with holed gear blanks

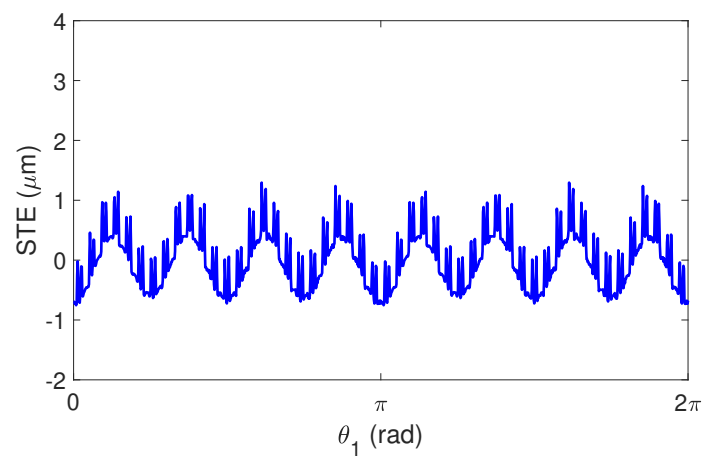

(a)

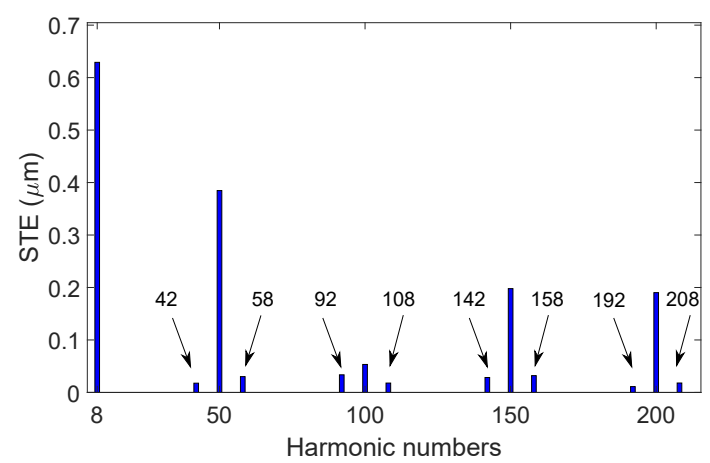

(b)

Figure 16: Fluctuation of the static transmission error of the gear pair with holes (a) and frequency spectrum (b) for an output torque $T=115 \mathrm{~N}$.m 


\section{Conclusion}

A flexible multibody approach through ANSYS Mechanical ${ }^{\circledR}$ solver is proposed to deal with gear mesh contact. The complete nonlinear problem is solved numerically by performing dynamic analysis at low rotating speed (1 rpm).

It was demonstrated that the investigated method can simulate large sort of gears. Besides, quasi-static numerical results of spur and helical gears showed differences in terms of static transmission and mesh stiffness compared with a classical approach. Indeed, a smoother transition from one tooth to another is identified. Then, the mean values and the fluctuations of the mesh stiffnesses are two times lower than those obtained with the classical approach. These results have significant influences on the vibroacoustic behaviour of gears.

Furthermore, the proposed methodology exhibits complex behaviours. For instance, Crowning modification increases the STE fluctuations. Flexible shafts and thin rim include misalignment, and therefore partial contact pressure distribution at the tooth flank. Holes in the gear blanks is adding non negligible harmonics components in the STE.

The proposed multibody approach provides a feasible methodology which can be extended to any types of gears (bevel gears, spiral gear, hypoid gears, worm gears etc.)

\section{Acknowledgements}

The authors would like to thanks ANSYS and the ANRT (French National Association for Research and Technology) for their support via a CIFRE grant. This work was performed within the framework of the LabCom LADAGE (LAboratoire de Dynamique des engrenAGEs), created by the LTDS and the Vibratec Company and operated by the French National Research Agency (ANR-14-LAB6- 0003). It was also performed within the framework of the LABEX CeLyA (ANR-10-LABX-0060) of Université de Lyon, within the program «Investissements d'Avenir » (ANR-16IDEX-0005) operated by the French National Research Agency (ANR). Special thanks go to A.Mélot from LTDS for the critical reading of the manuscript.

\section{Conflict of interest}

The authors declare that they have no conflict of interest.

\section{References}

[1] Andersson, A., Vedmar, L., 2003. A dynamic model to determine vibrations in involute helical gears. Journal of Sound and Vibration 260, 195-212.

[2] Cai, Y., 1995. Simulation on the Rotational Vibration of Helical Gears in Consideration of the Tooth Separation Phenomenon (A New Stiffness Function of Helical Involute Tooth Pair). Journal of Mechanical Design 117, 460-469. American Society of Mechanical Engineers Digital Collection.

[3] Carbonelli, A., Perret-Liaudet, J., Rigaud, E., Le Bot, A., 2011. Particle Swarm Optimization as an Efficient Computational Method in order to Minimize Vibrations of Multimesh Gears Transmission.

[4] Carbonelli, A., Rigaud, E., Perret-Liaudet, J., 2016. Vibro-Acoustic Analysis of Geared Systems-Predicting and Controlling the Whining Noise, in: Fuchs, A., Nijman, E., Priebsch, H.H. (Eds.), Automotive NVH Technology. Springer International Publishing, Cham. SpringerBriefs in Applied Sciences and Technology, pp. 63-79.

[5] Cescotto, S., Charlier, R., 1993. Frictional contact finite elements based on mixed variational principles. International Journal for Numerical Methods in Engineering 36, 1681-1701.

[6] Cescotto, S., Zhu, Y.Y., 1994. Large strain dynamic analysis using solid and contact finite elements based on a mixed formulation; application to metal forming. Journal of Materials Processing Technology 45, 657-663.

[7] Chen, Z., Shao, Y., 2011. Dynamic simulation of spur gear with tooth root crack propagating along tooth width and crack depth. Engineering Failure Analysis 18, 2149-2164.

[8] Chen, Z., Zhai, W., Shao, Y., Wang, K., 2016. Mesh stiffness evaluation of an internal spur gear pair with tooth profile shift. Science China Technological Sciences 59, 1328-1339.

[9] Conry, T.F., Seireg, A., 1973. A Mathematical Programming Technique for the Evaluation of Load Distribution and Optimal Modifications for Gear Systems. Journal of Engineering for Industry 95, 1115-1122.

[10] Fernández, A., Iglesias, M., de Juan, A., García, P., Sancibrián, R., Viadero, F., 2014. Gear transmission dynamic: Effects of tooth profile deviations and support flexibility. Applied Acoustics 77, 138-149.

[11] Garambois, P., Perret-Liaudet, J., Rigaud, E., 2017. NVH robust optimization of gear macro and microgeometries using an efficient tooth contact model. Mechanism and Machine Theory 117, 78-95.

[12] Greenwood, J.A., Williamson, J.B.P., Bowden, F.P., 1966. Contact of nominally flat surfaces. Proceedings of the Royal Society of London. Series A. Mathematical and Physical Sciences 295, 300-319. Royal Society.

[13] Guyan, R.J., 2012. Reduction of stiffness and mass matrices. AIAA Journal 
[14] Harris, S.L., 1958. Dynamic Loads on the Teeth of Spur Gears:. Proceedings of the Institution of Mechanical Engineers SAGE PublicationsSage UK: London, England.

[15] Houser, D.R., 1985. Gear Noise Sources and Their Prediction Using Mathematical Models. Gear Dynamics and Gear Noise Research Laboratory, Ohio State Univ.

[16] Johnson, K.L., 1987. Contact Mechanics. Cambridge University Press.

[17] Kahraman, A., Blankenship, G.W., 1999. Effect of Involute Tip Relief on Dynamic Response of Spur Gear Pairs. Journal of Mechanical Design 121, 313-315. American Society of Mechanical Engineers Digital Collection.

[18] Kocak, S., Akay, H.U., Ecer, A., 1999. Parallel Implicit Treatment of Interface Conditions in Domain Decomposition Algorithms, in: Lin, C.A., Ecer, A., Periaux, J., Satofuka, N., Fox, P. (Eds.), Parallel Computational Fluid Dynamics 1998. North-Holland, Amsterdam, pp. 353360.

[19] Koffel, G., Ville, F., Changenet, C., Velex, P., 2009. Investigations on the power losses and thermal effects in gear transmissions.

[20] Kurokawa, S., Ariura, Y., Ohtahara, M., 1996. Transmission errors of cylindrical gears under load - influence of tooth profile modification and tooth deflection. American Society of Mechanical Engineers, Design Engineering Division (Publication) DE 88, $213-217$.

[21] Laursen, T.A., Maker, B.N., 1995. An augmented Lagrangian quasi-Newton solver for constrained nonlinear finite element applications. International Journal for Numerical Methods in Engineering 38, 3571-3590.

[22] Mohan, Y., Seshaiah, T., 2012. Spur Gear Optimization By Using Genetic Algorithm. Int. J. Eng. Res. Appl 2, $311-318$.

[23] Munro, R., Yildirim, N., Hall, D., 1990. Optimum profile relief and transmission error in spur gears. Proceedings of Institution of Mechanical Engineers C , 35-42.

[24] Nevzat Özgüven, H., Houser, D.R., 1988. Mathematical models used in gear dynamics - a review. Journal of Sound and Vibration 121, $383-411$.

[25] Ničeno, B., Hanjalić, K., 2001. Large eddy simulation (LES) on distributed memory parallel computers using an unstructured finite volume solver, in: Jenssen, C.B., Andersson, H.I., Ecer, A., Satofuka, N., Kvamsdal, T., Pettersen, B., Periaux, J., Fox, P. (Eds.), Parallel Computational Fluid Dynamics 2000. North-Holland, Amsterdam, pp. 457-464.

[26] Opitz, H., Richards, E.J., 1968. A discussion on the origin and treatment of noise in industrial environments - Noise of gears. Philosophical Transactions of the Royal Society of London. Series A, Mathematical and Physical Sciences 263, 369-380. Royal Society.

[27] Parker, R.G., Vijayakar, S.M., Imajo, T., 2000. Non-linear dynamic response of a spur gear pair: modelling and experimental comparisons. Journal of Sound and Vibration 237, 435-455.

[28] Perret-Liaudet, J., 1996. An original method for computing the response of a parametrically excited forced system. Journal of Sound and Vibration 196, 165-177.

[29] Petrov, E.P., Ewins, D.J., 2003. Analytical Formulation of Friction Interface Elements for Analysis of Nonlinear Multi-Harmonic Vibrations of Bladed Disks. Journal of Turbomachinery 125, 364-371. American Society of Mechanical Engineers Digital Collection.

[30] Rigaud, E., Barday, D., 2007. Modelling and analysis of atatic aransmission arror. Effect of wheel body deformation and interactions between adjacent loaded teeth.

[31] Rémond, D., Velex, P., Sabot, J., 1993. Comportement dynamique et acoustique des transmissions par engrenages : synthèse bibliographique. CETIM.

[32] Sainsot, P., Velex, P., Duverger, O., 2004. Contribution of gear body to tooth deflections - A new bidimensional analytical formula. Journal of Mechanical Design, Transactions of the ASME 126, 748-752.

[33] Salles, L., Staples, B., Hoffmann, N., Schwingshack1, C., 2016. Continuation techniques for analysis of whole aeroengine dynamics with imperfect bifurcations and isolated solutions. Nonlinear Dynamics 86, 1897-1911.

[34] Sauer, R.A., 2011. Enriched contact finite elements for stable peeling computations. International Journal for Numerical Methods in Engineering 87, 593-616.

[35] Savsani, V., Rao, R.V., Vakharia, D.P., 2010. Optimal weight design of a gear train using particle swarm optimization and simulated annealing algorithms. Mechanism and Machine Theory 45, 531-541.

[36] Simo, J.C., Laursen, T.A., . An augmented lagrangian treatment of contact problems involving friction. Computers \& Structures 42.

[37] Suzuki, T., Umezawa, K., Houjoh, H., Bagiasna, K., 1986. Influence of Misalignment on Vibration of Helical Gear. Transactions of the Japan Society of Mechanical Engineers Series C 52, 1595-1600.

[38] Szwedowicz, J., Kissel, M., Ravindra, B., Kellerer, R., 2014. Estimation of Contact Stiffness and its Role in the Design of a Friction Damper.

[39] Tavakoli, M.S., Houser, D.R., 1986. Optimum Profile Modifications for the Minimization of Static Transmission Errors of Spur Gears. Journal of Mechanisms, Transmissions, and Automation in Design 108, 86-94. American Society of Mechanical Engineers Digital Collection.

[40] Ulaga, S., Ulbin, M., Flašker, J., 1999. Contact problems of gears using Overhauser splines. International Journal of Mechanical Sciences 41, 385-395.

[41] Umezawa, K., Sato, T., Ishikawa, J., 1984. Simulation on rotational vibration of spur gear.

[42] Umezawa, M., 1995. Effects of deviation of tooth surface errors of a helical gear pair on the transmission Eerror. Transactions of the Japan Society of Mechanical Engineers Series C 61, 3101-3107.

[43] Velex, P., Ville, F., 2009. An Analytical Approach to Tooth Friction Losses in Spur and Helical Gears-Influence of Profile Modifications. Journal of Mechanical Design 131.

[44] Vijayakar, S.M., . Contact analysis of gears using a combined finite element and surface integral method : gear technology july/august 1993.

[45] Vinayak, H., Singh, R., Padmanabhan, C., 1995. Linear dynamic analysis of multi-mesh transmissions containing external, rigid gears. Journal of Sound and Vibration 185, 1-32.

[46] Welbourn, D.B., 1979. Fundamental knowledge of gear noise: a survey. Number: IMechE-C117/79.

[47] Wriggers, P., 2006a. Discretization, Large Deformation Contact, in: Wriggers, P. (Ed.), Computational Contact Mechanics. Springer, Berlin, Heidelberg, pp. 225-307.

[48] Wriggers, P., 2006b. Discretization of the Continuum, in: Wriggers, P. (Ed.), Computational Contact Mechanics. Springer, Berlin, Heidelberg, 
On a flexible multibody modelling approach using FE-based contact formulation for describing gear transmission error

pp. 157-182.

[49] Wriggers, P., 2006c. Discretization, Small Deformation Contact, in: Wriggers, P. (Ed.), Computational Contact Mechanics. Springer, Berlin, Heidelberg, pp. 183-224.

[50] Wriggers, P., 2006d. Introduction to Contact Mechanics, in: Wriggers, P. (Ed.), Computational Contact Mechanics. Springer, Berlin, Heidelberg, pp. 11-29.

[51] Xiang, L., Gao, N., 2017. Coupled torsion-bending dynamic analysis of gear-rotor-bearing system with eccentricity fluctuation. Applied Mathematical Modelling 50, 569-584.

[52] Yang, D.C.H., Lin, J.Y., 1987. Hertzian Damping, Tooth Friction and Bending Elasticity in Gear Impact Dynamics. Journal of Mechanisms, Transmissions, and Automation in Design 109, 189-196. American Society of Mechanical Engineers Digital Collection.

[53] Yonekawa, K., Kawahara, M., 2001. Analysis of Tidal Flow Using Kalman Filter Finite Element Method with Domain Decomposition Method, in: Valliappan, S., Khalili, N. (Eds.), Computational Mechanics-New Frontiers for the New Millennium. Elsevier, Oxford, pp. 155-160.

[54] Yuruzume, I., Mizutani, H., Tsubuku, T., 1979. Transmission Errors and Noise of Spur Gears Having Uneven Tooth Profile Errors. Journal of Mechanical Design 101, 268-273. American Society of Mechanical Engineers Digital Collection.

\section{CRediT authorship contribution statement}

Y. Benaïcha: Conceptualization, Methodology, Software, Validation, Investigation, Writing - Original Draft, Writing - Review and Editing, Visualization. J. Perret-Liaudet: Conceptualization,Supervision, Funding acquisition, Writing - Review and Editing. J-D. Beley: Conceptualization,Supervision, Funding acquisition, Writing - Review and Editing. E. Rigaud: Supervision,Writing - Review and Editing. F. Thouverez: Conceptualization,Supervision, Funding acquisition, Writing - Review and Editing. 